\title{
EL EFECTO ASIMETRICO DEL CICLO ECONOMICO SOBRE LAS CONDICIONES SOCIALES ARGENTINAS EN EL PERÍODO 2003 - 2015
}

\author{
Guido Zack*
}

enviado: junio 2016 - aceptado: agosto 2016

\begin{abstract}
Resumen
Luego de la crisis de 2002, la Argentina inició un proceso de fuerte recuperación de sus condiciones sociales, que se desaceleró a partir de 2007 y se ha estancado desde 2012 en valores algo mejores en relación a la década de 1990, pero peores si la comparación se hace con décadas previas. A pesar del elevado crecimiento experimentado hasta 2011, la distribución del ingreso fue el principal causante de la mejora en la pobreza e indigencia. El presente artículo indaga acerca del riesgo de revertir en los próximos años parte de la recuperación conseguida. Este riesgo se sustenta en el posible efecto asimétrico del ciclo económico sobre las condiciones sociales, analizado a partir de las estimaciones de las elasticidades ingreso y distribución de la pobreza y la indigencia durante el período 2003 - 2015.
\end{abstract}

Códigos JEL: C22, E32, D31, I32

Palabras clave: Pobreza, crecimiento, distribución del ingreso, ciclo económico, Argentina.

\begin{abstract}
After the crisis of 2002, Argentina started a process of strong social condition recovery, which slowed from 2007 and has stagnated since 2012. Present situation is slightly better in relation to the 1990 s, but worse if the comparison is made with previous decades. Despite the high growth experienced until 2011, income distribution was the main cause of the improvement in poverty and extreme poverty. This article

* Instituto Interdisciplinario de Economía Política de Buenos Aires (IIEP - Baires)- Universidad de Buenos Aires - CONICET - Av. Córdoba 2122 2do (C1120 AAQ)- Ciudad Autónoma de Buenos Aires, República Argentina- +54 9114418 5462- zack.gui@gmail.com
\end{abstract}


investigates the risk of reversing in the coming years part of the recovery achieved. This risk is based on the possible asymmetric effect of the business cycle on social conditions, analyzed through the growth and income distribution elasticities of poverty and extreme poverty estimated for the $2003-2015$ period.

JEL Code: C22, E32, D31, I32

Keywords: Poverty, growth, income distribution, business cycle, Argentina.

\section{INTRODUCCION}

Tras la salida del régimen de Convertibilidad, la economía argentina se encontraba muy deteriorada. Entre mediados de 1998 y mediados de 2002, el producto interno bruto (PIB) se contrajo casi un $20 \%$, del cual más de la mitad era explicado por el último año. Esta evolución fue acompañada por un deterioro sin precedentes en las condiciones sociales. En efecto, la pobreza llegó a afectar en la segunda mitad de 2002 a más del $50 \%$ de la población, mientras que la indigencia superó el $23 \%$.

La historia posterior es bastante conocida. La combinación de un contexto internacional favorable con políticas internas expansivas permitió una fuerte y rápida recuperación, que posteriormente se convirtió en crecimiento económico. Este comportamiento se reflejó en las condiciones sociales, pues hacia 2011 la pobreza se ubicó por debajo del $20 \%$ de la población, mientras que la indigencia alcanzaba a menos del 5\%. Sin embargo, en los años posteriores, junto con la desaceleración de la actividad económica, las mejoras sociales se ralentizaron, hasta interrumpirse.

Este artículo indaga los motivos principales de la recuperación social en la Argentina y analiza los riesgos de revertir en los próximos años parte del progreso conseguido. Este riesgo se sustenta no solo en las dificultades que viene enfrentando el país para continuar con la senda del crecimiento económico, sino también en el posible efecto asimétrico del ciclo económico sobre las condiciones sociales. A partir de las estimaciones de las elasticidades ingreso y distribución de la pobreza y la indigencia, el presente artículo analiza si durante el periodo 2003 - 2015 un punto de crecimiento económico mejoró las condiciones sociales en la misma proporción que las deterioró un punto de caída del nivel de actividad. 
Para ello, el texto se divide en cinco partes. Luego de esta introducción, se hace un repaso de la literatura que trata el tema del efecto asimétrico del ciclo económico sobre la pobreza y la indigencia. En la tercera sección, se analiza la mejora de los indicadores sociales de la Argentina entre 2003 y 2015, diferenciando qué parte se debió al crecimiento económico y cuál a la distribución del ingreso. A continuación, se realiza un ejercicio econométrico en el que se estiman las elasticidades ingreso y distribución de la pobreza y, en particular, se testea si estas elasticidades muestran una magnitud similar según se trate de observaciones de incremento o caída del ingreso. Finalmente, se presentan las conclusiones.

\section{EL EFECTO ASIMETRICO DEL CICLO ECONOMICO SOBRE LOS INDICADORES SOCIALES}

Se considera pobre (indigente) a los miembros de toda familia cuyo ingreso por adulto equivalente no supere la línea de pobreza (indigencia). Así, la cantidad de pobres puede disminuir por dos efectos: el crecimiento promedio de la economía y la distribución del ingreso.

Sin embargo, a mediados del siglo pasado, un artículo marcaría la discusión acerca del efecto del crecimiento sobre la pobreza, al indicar que la distribución del ingreso tendía a deteriorarse durante los primeros estadios de desarrollo de un país, hasta cierto punto en el que la expansión comenzaba a ser distribuida en forma más igualitaria (Kuznets, 1955). Esta teoría fue denominada "U invertida", por la forma en la que se veía la desigualdad a medida que una economía se desarrollaba. De este modo, si el crecimiento económico puede generar una distribución del ingreso menos igualitaria, su efecto final sobre la pobreza es indeterminado.

Si bien el mismo Kuznets fue cauto en relación a sus conclusiones, muchos autores defendieron su hipótesis (Kravis 1960, Oshima 1970, Adelman y Morris 1973, Paukert 1973, Cline 1975, Ahluwalia 1976, Robinson 1976, entre otros). No obstante, estas investigaciones utilizaron datos de corte transversal, cuando realmente sería necesario contar con la dimensión temporal para testear correctamente la hipótesis (Fields, 1989). De hecho, investigaciones posteriores que hicieron uso de series temporales (Ahluwalia 1974, Fields 1980, Ram 1991) o paneles (Partridge et ál. 1996, Bruno et ál. 1998) no encontraron evidencia de una relación inversa entre crecimiento y distribución.

Incluso, autores como de Janvry y Sadoulet (2000), Lustig (2000) y Agénor (2004), entre otros, hallaron que el crecimiento y la igualdad se relacionan en for- 
ma directa, pero asimétrica. Esto quiere decir que durante períodos de crecimiento la distribución del ingreso tiende a mejorar, mientras que en fases descendentes del ciclo la desigualdad aumenta. Pero las variaciones no son simétricas, sino que la mejora durante el auge es más débil que el deterioro en las recesiones. De esta manera, la volatilidad real afectaría negativamente la distribución del ingreso (Hausmann y Gavin 1996, Laursen y Mahajan 2005, Breen y García-Peñalosa 2005, Calderón y Levy Yeyati 2009, Fang et al. 2015).

En definitiva, si el ciclo económico tiene un efecto asimétrico sobre la distribución del ingreso, entonces este efecto necesariamente tiene que verse reflejado en las variaciones de la pobreza y la indigencia. Al respecto, el BID (1995: 203 - 207) sostiene que si América Latina hubiera tenido el nivel de volatilidad macroeconómica de los países desarrollados, en 1995 su pobreza habría sido un $25 \%$ menor. En lo que sigue se analiza la existencia de este efecto asimétrico del ciclo económico sobre los indicadores sociales para el caso argentino entre 2003 y 2015. Para ello, a continuación se observa la evolución de los indicadores en el período, para luego proceder a testear la existencia de la asimetría a través de la estimación de las elasticidades crecimiento y distribución de la pobreza y la indigencia.

\section{LA RECUPERACION SOCIAL DE LA ARGENTINA LUEGO DE LA CONVERTIBILIDAD}

\section{II.1. La tasa, brecha y brecha al cuadrado de la pobreza y la indigencia}

A mediados de 2003, la Argentina se encontraba recuperándose de la peor crisis económica de su historia. La pobreza alcanzaba a casi a la mitad de la población, mientras que la indigencia afectaba a más de la quinta parte. En los años subsiguientes, el fuerte crecimiento económico y la mejora en la distribución del ingreso permitieron una rápida recuperación de las condiciones sociales. En efecto, en el tercer trimestre de 2006 , la pobreza se ubicaba en torno al $25 \%$ de la población y la indigencia había perforado el piso del 10\%. Si bien las mejoras continuaron hasta $2013^{1}$, el ritmo se desaceleró, lo que habría sido consecuencia del efecto conjunto del aumento en la inflación y de la mayor precariedad estructural de la población pobre. Finalmente, en 2014 se observó el primer deterioro significativo del período, producto de la caída del ingreso real y del deterioro en la

1 A partir de diciembre de 2006 los valores de las canastas básicas de alimentos (CBA; línea de indigencia) y total (CBT; línea de pobreza) se actualizaron mediante el IPC -9 provincias. 
distribución como resultado de la devaluación de enero, que fue solo parcialmente revertido en los dos primeros trimestres de 2015 (Tabla 1).

Pero las tasas de pobreza e indigencia no son los únicos datos relevantes para analizar las condiciones sociales de una economía. En efecto, estos indicadores captan la incidencia (población pobre o indigente como porcentaje de la total), pero no la profundidad (cuán lejos están las familias pobres o indigentes de dejar de serlo). Una forma de dar cuenta de la profundidad es a través de la brecha de pobreza (indigencia), entendida como la diferencia entre la CBT (CBA) y el ingreso familiar por adulto equivalente de las familias pobres (indigentes). Sumando las brechas de pobreza (indigencia) de todos los miembros de las familias pobres (indigentes) se obtiene una idea de la profundidad del fenómeno.

Figura 1. Tasas de pobreza e indigencia de la Argentina (\% de la población)

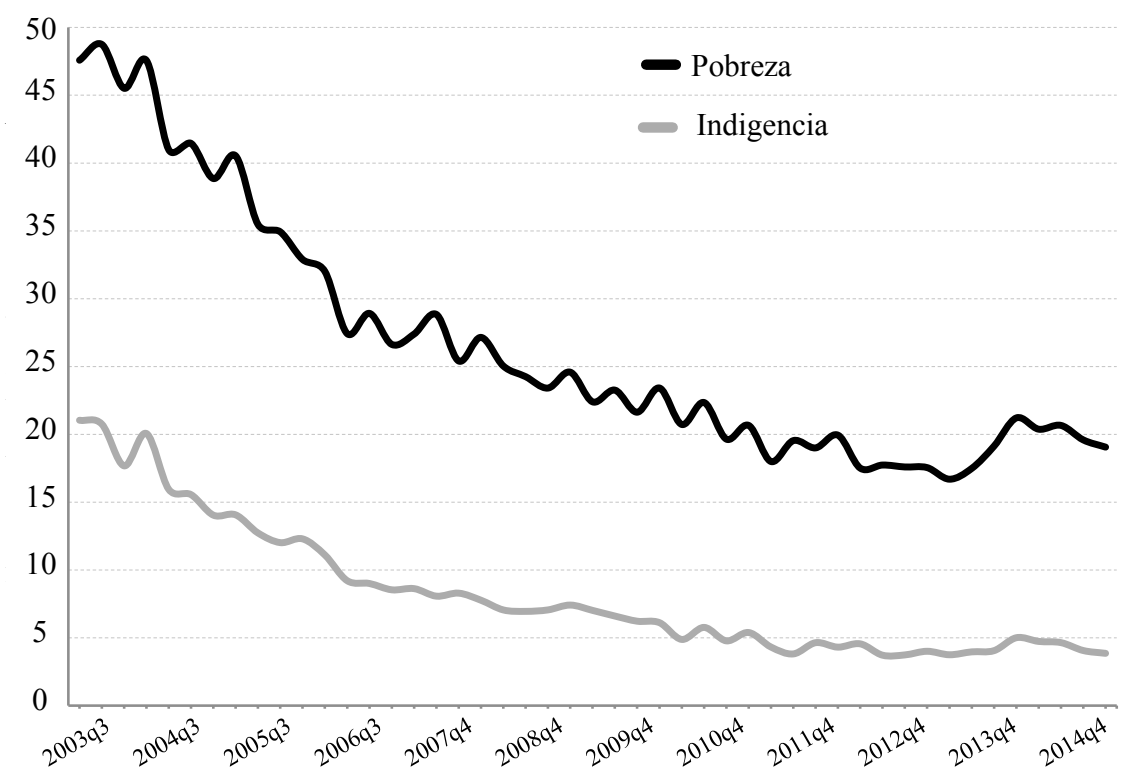

la pobreza e indigencia en términos del ingreso total de la población se hace con el ingreso de los estratos más altos, se obtiene que al segundo trimestre de 2015 era necesaria una transferencia del 5,5\% del ingreso del quinto quintil para eliminar la pobreza y del $0,6 \%$ para hacer lo propio con la indigencia. Estos valores ascienden a $8,5 \%$ y $1 \%$, respectivamente, si la transferencia proviene del décimo decil. 
Figura 2. Brecha de pobreza e indigencia de la Argentina ( $\%$ del ingreso total)

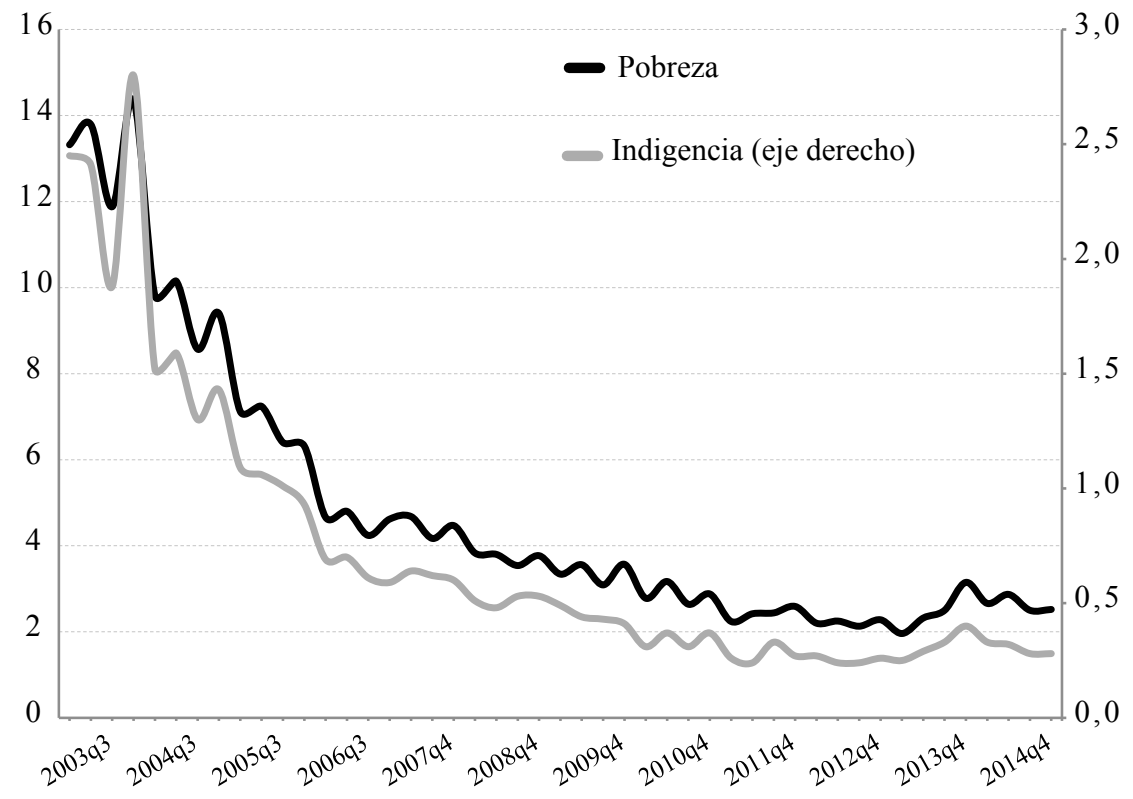

Fuente: Elaboración propia con base en microdatos EPH - INDEC

Entonces, las brechas corrigen a las tasas en que no solo tienen en cuenta la incidencia del problema sino también la profundidad. Sin embargo, ninguno de los dos indicadores considera la distribución del ingreso al interior de la población pobre o indigente ${ }^{2}$. Una forma de considerarla es a través de la brecha al cuadrado de la pobreza, indicador que otorga al individuo pobre una ponderación mayor cuanto menor sea su ingreso. Para el caso de Argentina entre 2003 y 2015, las brechas al cuadrado de la pobreza e indigencia mostraron una evolución similar a las tasas y a las brechas. La diferencia principal es que el deterioro comenzó un año antes, es decir, en 2012 en lugar de 2013, lo que da cuenta de que la distribución del ingreso al interior de la población pobre e indigente mostró un deterioro en dicho año (Figura 3).

2 Por ejemplo, la brecha de pobreza es idéntica en el caso de que todos los pobres tengan un ingreso equivalente al $50 \%$ de la canasta básica total (y por lo tanto la distribución del ingreso al interior de la población pobre sea totalmente igualitaria) que en el caso de que la mitad tenga un ingreso equivalente al $90 \%$ de la canasta y el resto al $10 \%$. 
Figura 3. Brecha al cuadrado de pobreza e indigencia de la Argentina (canastas básicas al cuadrado por habitante)

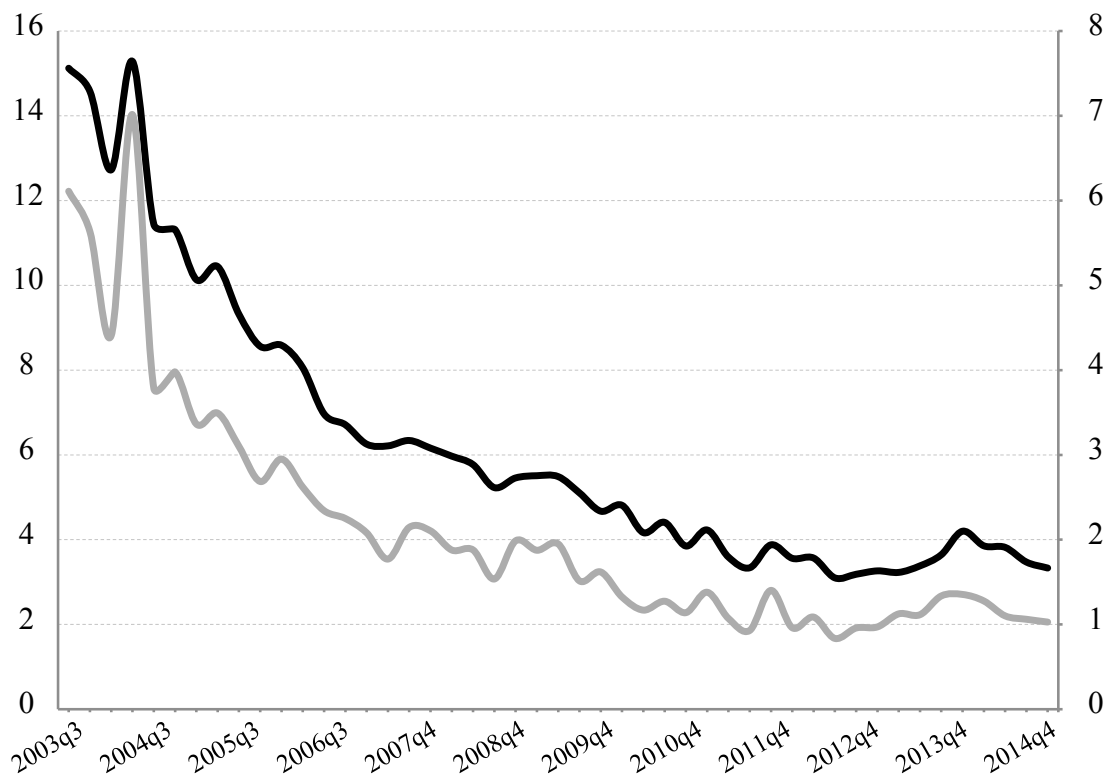

Fuente: Elaboración propia con base en microdatos EPH - INDEC

En definitiva, entre 2003 y 2015 las condiciones sociales mostraron una importante recuperación en la Argentina. Sin embargo, esta recuperación no representa una mejora sustancial en relación a los valores presentados en décadas pasadas. En efecto, si bien la metodología de la EPH fue modificada en 2003 y la comparabilidad se ve afectada, la tasa de pobreza en el primer semestre de 1998 fue de $18,1 \%$ de la población, es decir, por debajo de la del segundo trimestre de 2015 y no muy por encima del mínimo observado en el tercer trimestre de 2013. No obstante, la tasa de indigencia mostró una mejora más sustancial, ya que los valores en torno a 4\% que se observan desde 2011 nunca habían sido alcanzados en la década de 1990. Lo mismo sucedió con las brechas y brechas al cuadrado de pobreza e indigencia. Pero, en cualquier caso, tanto la incidencia como la profundidad de la pobreza y la indigencia se encuentran hoy muy por encima de los registros observados hasta finales de los ' $80 \mathrm{~s}$. 


\section{II.2. Efecto crecimiento y efecto distribución}

Como se mencionó previamente, los indicadores de pobreza varían básicamente por dos fenómenos diferenciables: el crecimiento y la distribución del ingreso. Es por eso que resultó interesante hacer esta descomposición para el caso argentino en el período de estudio. De esta forma, no solo se puede analizar cuál de estos efectos fue el más importante para explicar la recuperación de las condiciones sociales en el país entre 2003 y 2015, sino que las series desagregadas se van a utilizar como ejercicio de robustez en las estimaciones de las elasticidades ingreso y distribución de la pobreza y la indigencia.

Si bien hay numerosos métodos para hacer esta descomposición (Medina y Galván 2014), en este caso se optó por utilizar el desarrollado por Mahmoudi (2001). El mismo es similar al propuesto por Datt y Ravallion (1992), pero con la diferencia que los efectos crecimiento y distribución se computan promediando los resultados que surgen de fijar como referencia los períodos inicial y final. Así, es un método de descomposición exacto (no existe residuo) y no es sensible al período de referencia (Medina y Galván 2014, pp. 24-26). Asimismo, este método fue utilizado previamente para la Argentina y otros países de América Latina, aunque para períodos diferentes (Busso et al. 2005, Gasparini et ál. 2007, Gasparini et ál. 2013 pp. 554-561, entre otros).

En lo que sigue, entonces, se analiza la contribución de estos dos efectos a la recuperación de los indicadores sociales en la Argentina entre 2003 y 2015. Como puede verse en las Tablas 1 y 2 , excepto para la brecha al cuadrado de la indigencia, en todos los indicadores el efecto crecimiento fue el factor más importante para explicar las mejoras al inicio del período, pero desde mediados de 2006 ha ido perdiendo relevancia. En la comparación punta a punta, es decir, entre el tercer trimestre de 2003 y el segundo de 2015, el crecimiento explica 15,5 puntos porcentuales (p.p.) de un total de 28,5 p.p. de caída en la tasa de pobreza (lo que representa el $54,4 \%$ del total), quedando los 13 p.p. $(45,6 \%)$ restantes para la distribución. A medida que se va pasando a los indicadores de brecha, el efecto crecimiento va perdiendo poder explicativo, en detrimento del distribución. Así, de la caída de 17,1 p.p. de la brecha de pobreza y de 11,8 p.p. de la brecha al cuadrado, 7,2 p.p. $(42,1 \%)$ y 4,3 p.p. $(36,4 \%)$ son consecuencia del aumento en el ingreso, mientras que 9,9 p.p. $(57,9 \%)$ y 7,5 p.p. $(63,6 \%)$, respectivamente, de la distribución (Tabla 1). 
EL EFECTO ASIMETRICO DEL CICLO ECONOMICO SOBRE LAS CONDICIONES SOCIALES...

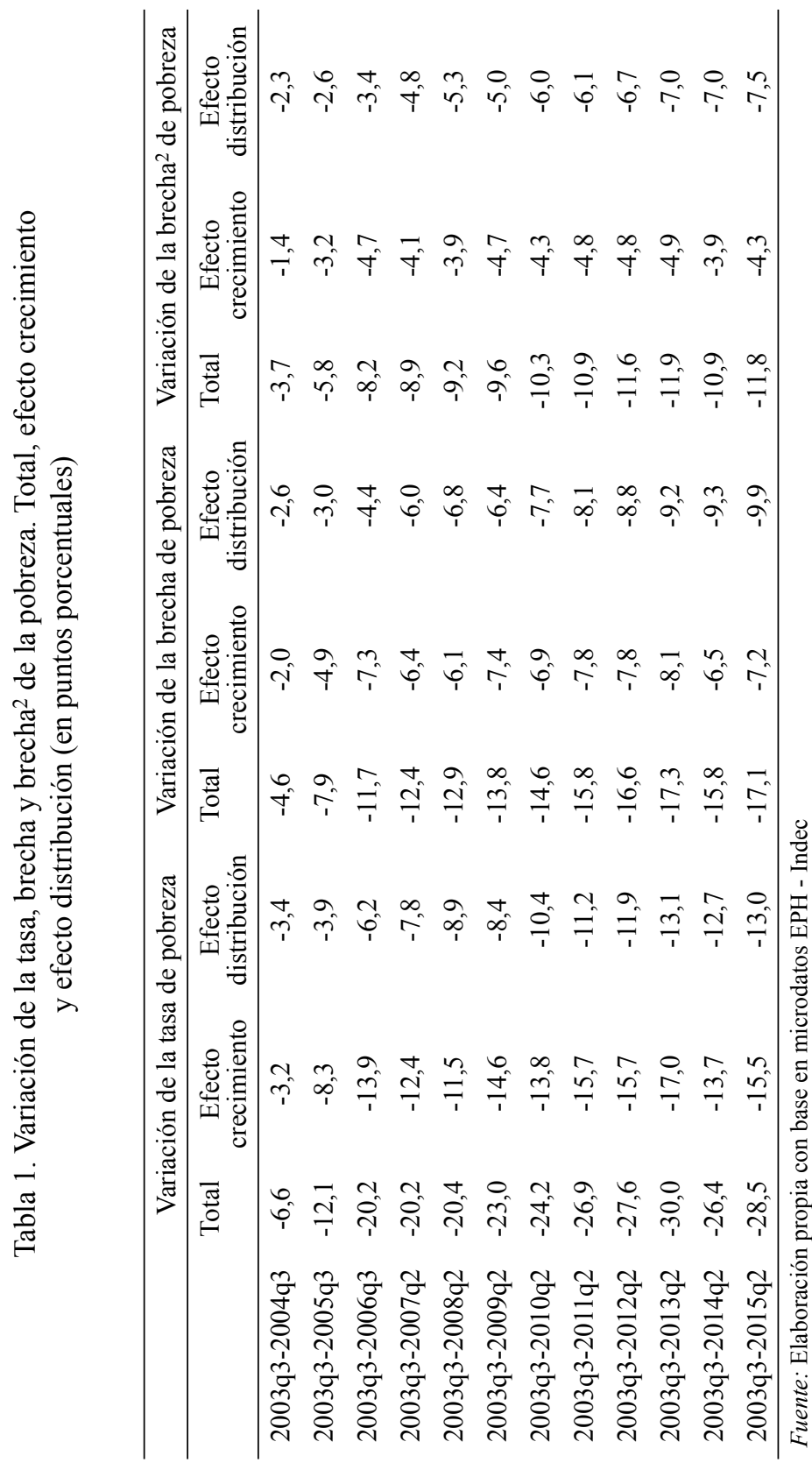


Para todos los indicadores de indigencia, el crecimiento es un factor explicativo de menor importancia en comparación a los indicadores de pobreza. Así, de 17,2 p.p. de la variación negativa de la tasa, 8 p.p. de la brecha y 5,1 p.p. de la brecha al cuadrado, 5,7 p.p. $(33,1 \%), 2,3$ p.p. $(28,75 \%)$ y 1,3 p.p. $(25,5 \%)$, respectivamente, son explicados por el aumento del ingreso, mientras que los restantes 11,5 p.p. $(66,9 \%), 5,7$ p.p. $(71,25 \%)$ y 3,8 p.p. $(74,5 \%)$, respectivamente, por la distribución. Como puede notarse, en todos los indicadores de indigencia la distribución es el factor más relevante. Asimismo, se da el mismo patrón que en la pobreza, por el cual a medida que se pasa de la tasa a la brecha y a la brecha al cuadrado, la importancia de la distribución aumenta, en detrimento del crecimiento (Tabla 2).

Entonces, entre 2003 y 2015, incluso habiendo sido un período de elevado crecimiento (al menos hasta 2011), la distribución del ingreso fue el efecto más importante en la mejora de la mayor parte de los indicadores sociales ${ }^{3}$. Al respecto, el índice de Gini mostró una notable mejora entre 2003 y 2012, para luego estabilizar su tendencia aunque con bastante volatilidad. Este comportamiento se observa no solo al tomar los ingresos de toda la población, sino también en el caso de considerar únicamente los ingresos de los pobres (Figura 4). Justamente, la mejora en la distribución del ingreso al interior de la población pobre es la causante de que el efecto distribución sea un factor más relevante para explicar la caída en las brechas al cuadrado en relación a las brechas y a las tasas.

En resumen, la Argentina ha logrado una significativa recuperación en sus condiciones sociales, aunque aún mantiene niveles de pobreza e indigencia elevados en términos históricos. El período analizado puede ser dividido, al menos, en tres partes. La primera, entre 2003 y 2006, en la que la mejora fue muy intensa, liderada por el crecimiento económico. La segunda, entre 2007 y 2011, la caída en la pobreza y la indigencia continuó, aunque a menor ritmo y liderada por la distribución del ingreso. La tercera, de 2012 a la fecha, con importantes fluctuaciones, el crecimiento y la mejora en la distribución del ingreso se estancaron, por lo que también se detuvo la disminución de la pobreza y la indigencia, que incluso aumentaron levemente.

3 Este resultado se encuentra en línea con los hallados por Gasparini et al. (2007) y Madina y Galván (2014, pp. 71-72) para la Argentina y otros países de América Latina, aunque para períodos diferentes. 


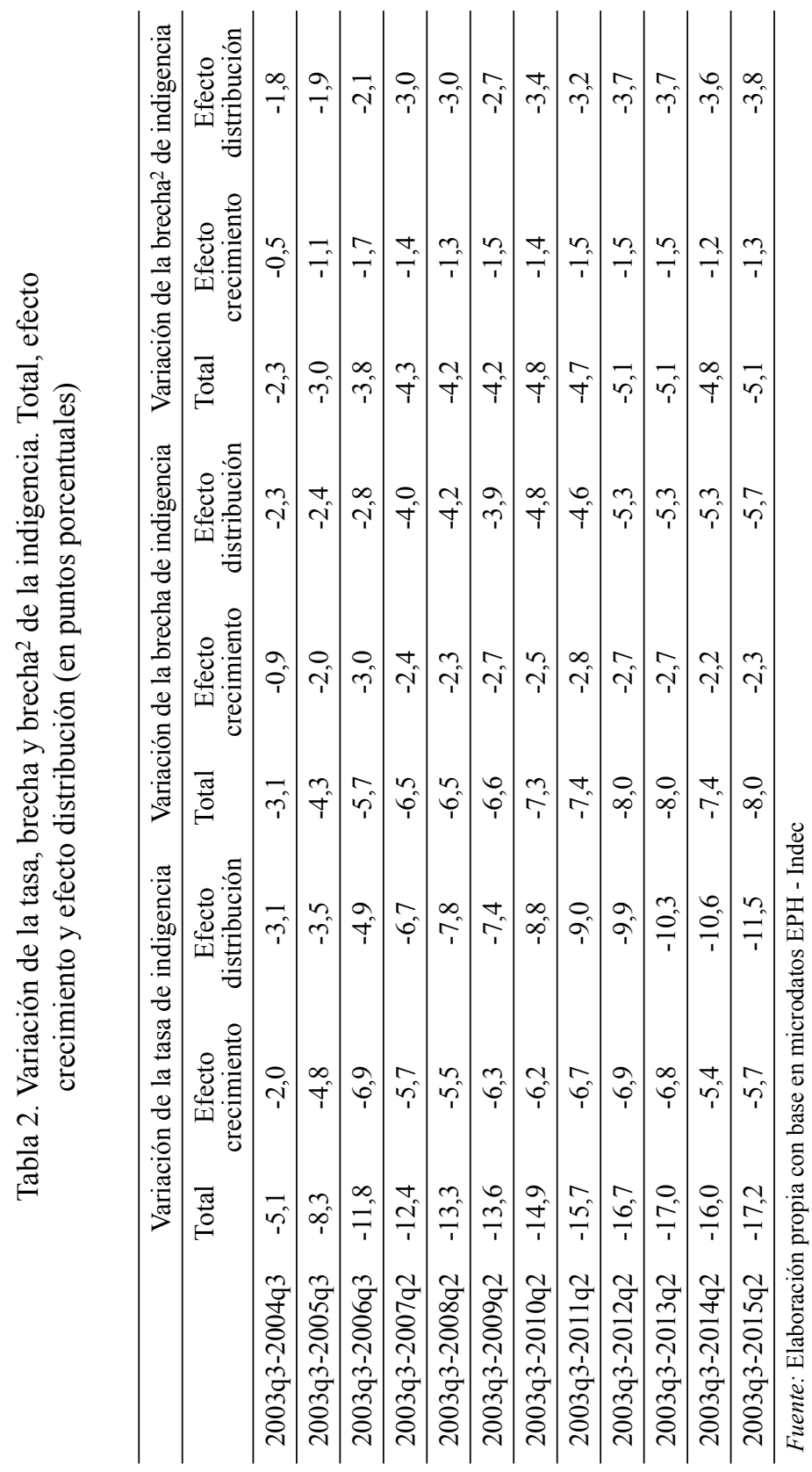


Figura 4. Índice de Gini

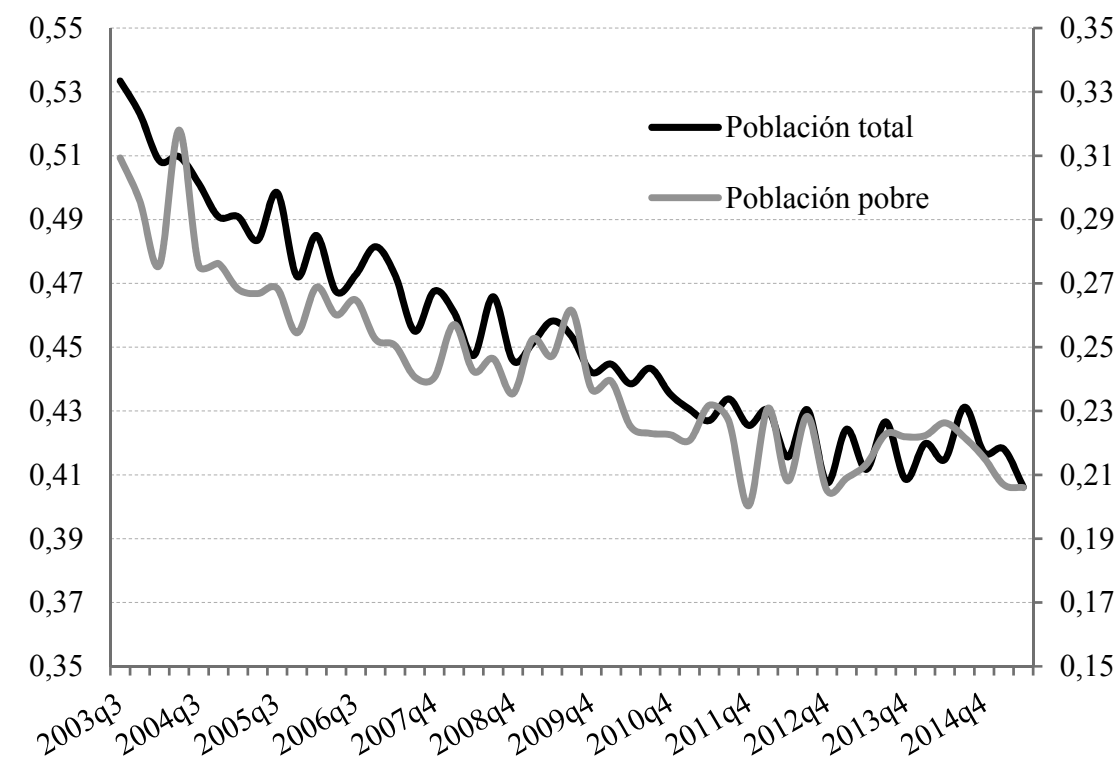

Fuente: Elaboración propia con base en microdatos EPH - INDEC

\section{LA ASIMETRIA EN LA ELASTICIDAD INGESO DE LA POBREZA E INDIGENCIA}

Hasta aquí se ha visto que hasta 2012 Argentina había mostrado una importante recuperación en sus condiciones sociales. No obstante, retomar dicha tendencia depende, entre otras cosas, de alcanzar un crecimiento económico, el cual se ve dificultado por un contexto internacional menos favorable y una situación cambiaria muy inestable. Así, se plantea la duda acerca de la posibilidad de mantener la presente situación social, en particular, en caso de existir un efecto asimétrico del ciclo sobre la pobreza y la indigencia.

Para evaluar la pertinencia de esta afirmación, en lo que sigue se estiman las elasticidades ingreso y distribución de la pobreza e indigencia para el período 2003 - 2015. También se hace el ejercicio para la brecha y la brecha al cuadrado. Asimismo, se testea si el efecto de las variaciones positivas del ingreso sobre los 
indicadores sociales es de magnitud similar al efecto de las variaciones negativas ${ }^{4}$. En lo que sigue se explica brevemente la metodología utilizada, para luego proceder a mostrar los resultados obtenidos.

\section{III.1. Metodología}

La metodología utilizada consiste en un modelo de corrección de error. Este modelo es muy usual para relacionar variables integradas de orden 1, las cuales suelen presentar relaciones de equilibrio. Consiste en un procedimiento en dos etapas. En la primera, se hace una regresión entre las variables en niveles o logaritmos, de forma de detectar las relaciones de largo plazo. De esta ecuación se extrae el residuo y se analiza su orden de integración. En caso de que el residuo sea estacionario, es decir, integrado de orden 0 , se concluye la existencia de relaciones de cointegración entre las variables. Esto se debe a que la combinación lineal de variables integradas de orden 1 da como resultado una variable con un orden menor de integración. En ese caso, el segundo paso consiste en regresar las variables en diferencias, por lo que ya serían estacionarias, incluyendo como variable explicativa a la serie de residuos de la ecuación de largo rezagada un período. Esta variable, denominada término de corrección de error, mide justamente qué porcentaje del desvío de la relación de largo plazo se corrige en cada período. El resultado del segundo paso son las relaciones de corto plazo de las variables.

En el caso específico del presente artículo, se desarrolla un modelo de corrección de error para cada una de las variables dependientes, es decir, la cantidad de pobres e indigentes, así como la brecha y la brecha al cuadrado de la pobreza y la indigencia ${ }^{5,6}$. La ecuación de largo plazo (1) relaciona al logaritmo natural de cada una de estas variables con el logaritmo del ingreso familiar por adulto equi-

4 Algunos trabajos, como Ravallion y Chen (1997), de Janvry y Sadoulet (2000), Bourguignon (2003), Adams (2004) y Gasparini et ál. (2007), entre otros, ya estimaron estas elasticidades, aunque sin testear la asimetría.

5 Cabe aclarar que, de forma que sean integradas de orden 1 y pueda utilizarse esta metodología, como indicador de pobreza e indigencia se tomó la cantidad de individuos en dicha situación, mientras que para las brechas se consideró la cantidad total de canastas básicas necesarias para eliminar la pobreza y la indigencia.

6 Como señalan Locke Anderson (1964), Bhalla (2002) y Gasparini ét al. (2007), el valor de las elasticidades ingreso y distribución de la pobreza e indigencia son muy sensibles a la ubicación de la CBT y CBA en la distribución del ingreso y a la magnitud de la tasa de crecimiento. En cambio, en el caso de la brecha y brecha al cuadrado, este efecto se ve reducido en forma muy significativa. 
valente ${ }^{7}$ y del índice de Gini. También se agrega una variable dicotómica que toma valor 1 para todos los trimestres cuya variación interanual del ingreso haya sido negativa ${ }^{8}$. Además, esta variable dicotómica es multiplicada por las dos variables independientes, de forma de identificar si las elasticidades presentan una magnitud diferente según se trate de un período de crecimiento o decrecimiento del ingreso. Lo que se busca básicamente es identificar el efecto asimétrico del ciclo económico sobre los indicadores de pobreza e indigencia. La ecuación de corto plazo (2), relaciona las mismas variables, pero en este caso transformadas a través de la diferencia del logaritmo. En esta ecuación se incluye al residuo de la ecuación de largo plazo retardado un periodo, es decir, el término de corrección de error.

$$
\begin{gathered}
\ln P_{t}=\alpha_{0}+\alpha_{1} \ln Y_{t}+\alpha_{2} \ln G_{t}+\alpha_{3} D+\alpha_{4} D \ln Y_{t}+\alpha_{5} D \ln G_{t} \\
+\alpha_{6} T+\varepsilon_{t} \\
\begin{array}{c}
\Delta \ln P_{t}=\beta_{0}+ \\
+\beta_{1} \Delta \ln Y_{t}+\beta_{2} \Delta \ln G_{t}+\beta_{3} D+\beta_{4} D \Delta \ln Y_{t} \\
+\beta_{5} D \Delta \ln G_{t}+\gamma \varepsilon_{t-1}+\epsilon_{t}
\end{array}
\end{gathered}
$$

Donde $\mathrm{P}_{\mathrm{t}}$ es el indicador de pobreza o indigencia, $\mathrm{Y}_{\mathrm{t}}$ es el ingreso familiar por adulto equivalente, $G_{t}$ es el índice de Gini, $T$ es la tendencia, $D$ es la variable dicotómica, $\alpha_{\mathrm{i}}$ son las elasticidades de largo plazo, $\beta_{\mathrm{i}}$ son las elasticidades de corto plazo, $\gamma$ es el factor de corrección de error, ln es el logaritmo neperiano y $\Delta$ es el operador de primeras diferencias.

Como se ha visto en la sección anterior, la variación de los tres indicadores de pobreza e indigencia pueden ser desagregados a partir del efecto crecimiento y distribución. Por lo tanto, el ejercicio planteado recientemente se realiza no solo para la variación total de los indicadores, sino también para la variación resultante únicamente del crecimiento del ingreso, por un lado, y del efecto distribución, por el otro. Claro está que la única variable explicativa en el primer caso es el ingreso, mientras que en el segundo solo se utiliza al índice de Gini.

7 El ingreso es considerado en términos reales. Para ello, se deflactó el ingreso nominal por adulto equivalente promedio del período por la CBT para el caso de las ecuaciones de pobreza y por la CBA para las de indigencia.

8 La variación interanual del ingreso real entre el segundo trimestre de 2003 y el segundo trimestre de 2015 fue negativa en 10 ocasiones, por lo que la variable dicotómica toma valor 1 en 10 observaciones de un total de 42 . 


\section{III.2. Resultados}

Todas las series tienen periodicidad trimestral y abarcan el período $3^{\circ}$ trimestre de $2003-2^{\circ}$ trimestre de 2015, es decir toda la serie disponible de la Encuesta Permanente de Hogares (EPH) en su modalidad continua.

Como se desprende de la metodología, el primer paso es analizar el orden de integración de las variables. Para ello, como se muestra en la Tabla A.1 del apéndice, se realizaron los contrastes de raíces unitarias a través del test de Dickey-Fuller (1981) y en ningún caso se puede rechazar la hipótesis nula de raíz unitaria para los niveles de las variables (en logaritmos). En cambio, los contrastes de raíz unitaria de los residuos de las ecuaciones de largo plazo dieron como resultado el rechazo de la hipótesis nula de raíz unitaria, por lo que se pueden considerar estacionarios ${ }^{9}$ (TablaA.2). De esta manera, se puede suponer la existencia de relaciones de cointegración entre las variables. Por último, la Tabla A.3 del apéndice muestra, a través del estadístico de Ljung-Box (1978), que no hay evidencia para rechazar la hipótesis nula de que los residuos de todas las ecuaciones de corto plazo sean ruido blanco, por lo que se admite la adecuación del modelo de corrección de error.

En el Tabla 3 se presentan las elasticidades estimadas para la cantidad de pobres, la brecha y la brecha al cuadrado para el período 2003 - 2015. Como puede observarse, en términos generales, las variables evidencian un buen nivel de significatividad y el signo es el esperado. Tanto para el largo como para el corto plazo, las elasticidades ingreso de la pobreza, la brecha y la brecha al cuadrado son negativas y sus valores absolutos se ubican apenas por encima de la unidad. Esto quiere decir que el crecimiento del $1 \%$ del ingreso generó una reducción algo mayor al $1 \%$ en los tres indicadores de pobreza, a la vez que el efecto tendió a completarse rápidamente habida cuenta de la similitud entre las elasticidades de corto y largo plazo. Por el lado de la elasticidad distribución, la magnitud muestra una mayor dispersión y aumenta a medida que se pasa de la cantidad de pobres a la brecha y a la brecha al cuadrado. Este comportamiento se explica porque el progreso en la distribución del ingreso impacta en la cantidad de pobres solo si los ingresos superan la CBT, en cambio la brecha y, más aún, la brecha al cuadrado mejoran incluso si los ingresos se mantienen por debajo de la línea de pobreza.

Por su parte, el coeficiente que acompaña al producto del ingreso y la variable dicotómica es significativo en muchos casos y su signo es negativo. Esto daría

9 Este resultado se alcanzó incluso utilizando un criterio más exigente de rechazo, como son los valores críticos de MacKinnon (1991). 
a entender que el valor absoluto de la elasticidad ingreso de la pobreza es mayor en los períodos de caída del ingreso que en momentos de crecimiento. En otras palabras, hay evidencia de la existencia de un efecto asimétrico del ciclo económico sobre la pobreza entre 2003 y 2015. En cambio, no parece haber diferencia entre el valor de la elasticidad distribución de la pobreza según se trate de una fase ascendente o descendente del ciclo. Esto se debe a que el único caso en el que el índice de Gini presenta un quiebre es cuando se considera la variación de la cantidad de pobres solo por el efecto distribución en el corto plazo.

En la Tabla 4 se presentan los resultados de las ecuaciones para la cantidad de indigentes, brecha y brecha al cuadrado, a partir de los cuales se alcanzan conclusiones similares que para el caso de la pobreza. En efecto, puede verse que la elasticidad ingreso tanto de corto como de largo plazo se ubican en las cercanías de la unidad. Asimismo, la elasticidad distribución muestra un valor y una dispersión superior. Es interesante notar también que la elasticidad distribución de la indigencia es bastante mayor que la de la pobreza. Esto es consistente con los datos observados en la sección anterior, en los que el efecto distribución era más relevante para la reducción de la indigencia que para la caída de la pobreza. Por su parte, al igual que en el caso de la pobreza, la elasticidad distribución crece a medida que se pasa de la indigencia a la brecha y brecha al cuadrado. La explicación es la misma y se relaciona con las transferencias de los no indigentes a los indigentes que, si bien en algunos casos no alcanzaron para sacar a la población de la indigencia, sí les permitió mejorar su situación y reducir la brecha.

Finalmente, también se encuentra evidencia del efecto asimétrico del ciclo económico sobre la indigencia entre 2003 y 2015, aunque en este caso hay que hacer algunas aclaraciones adicionales. En primer lugar, se identifica un quiebre en la elasticidad ingreso de la indigencia en ocho de los doce casos estimados. Sin embargo, en dos de ellos (efecto crecimiento de largo plazo para la brecha y la brecha al cuadrado) el valor del coeficiente es muy cercano a cero. Como contrapartida, en el caso de largo y corto plazo del efecto total de la brecha al cuadrado, los p-valores de la variable se ubican apenas por encima del 10\% (14,5\% y $11,2 \%)$. En definitiva, hay evidencia del efecto asimétrico, aunque algo más débil que en el caso de la pobreza. Por el lado de la elasticidad distribución, en línea con los resultados alcanzados para la pobreza, en ninguno de los casos se identifica que la magnitud dependa de la fase del ciclo económico. 
EL EFECTO ASIMETRICO DEL CICLO ECONOMICO SOBRE LAS CONDICIONES SOCIALES...

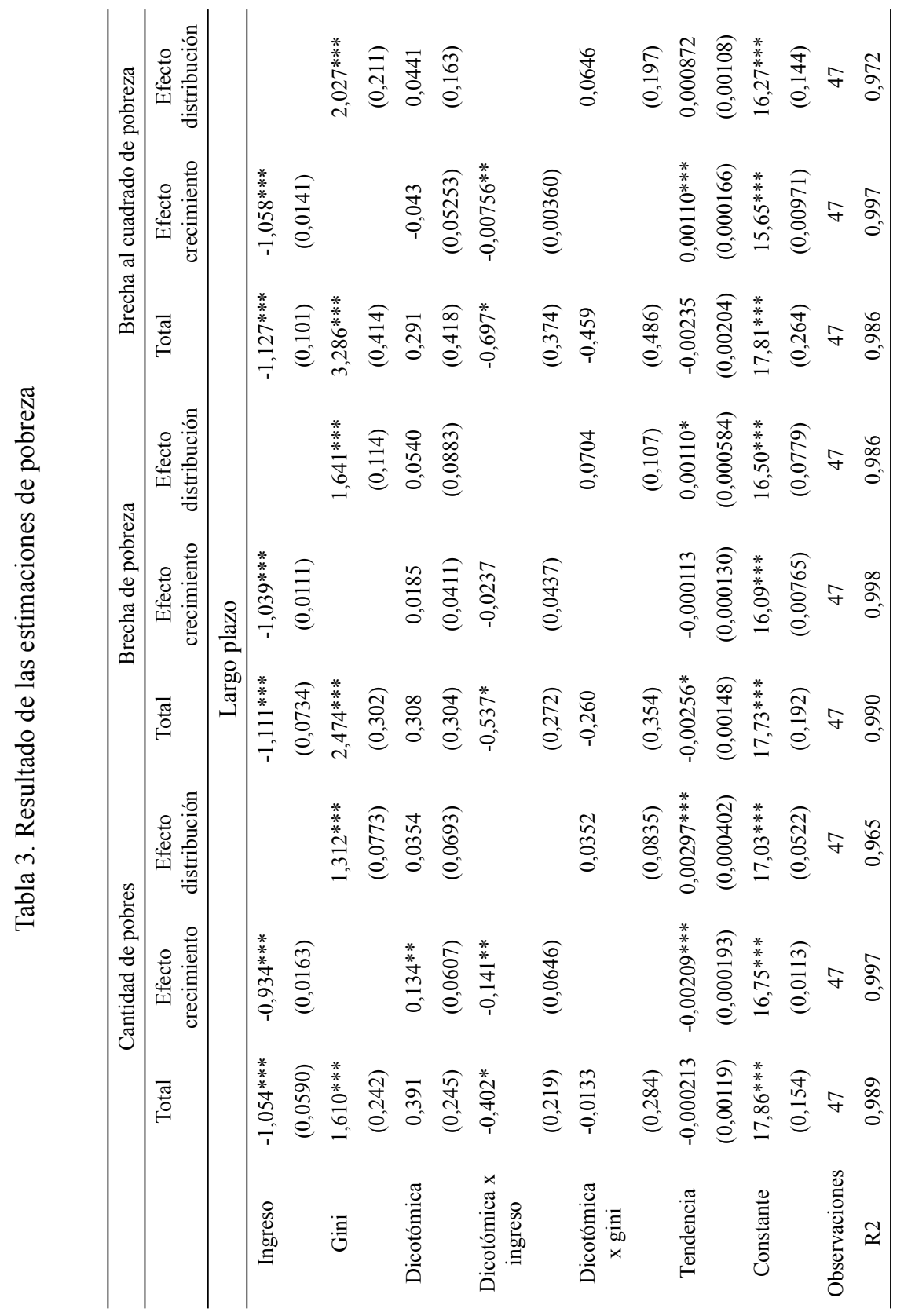




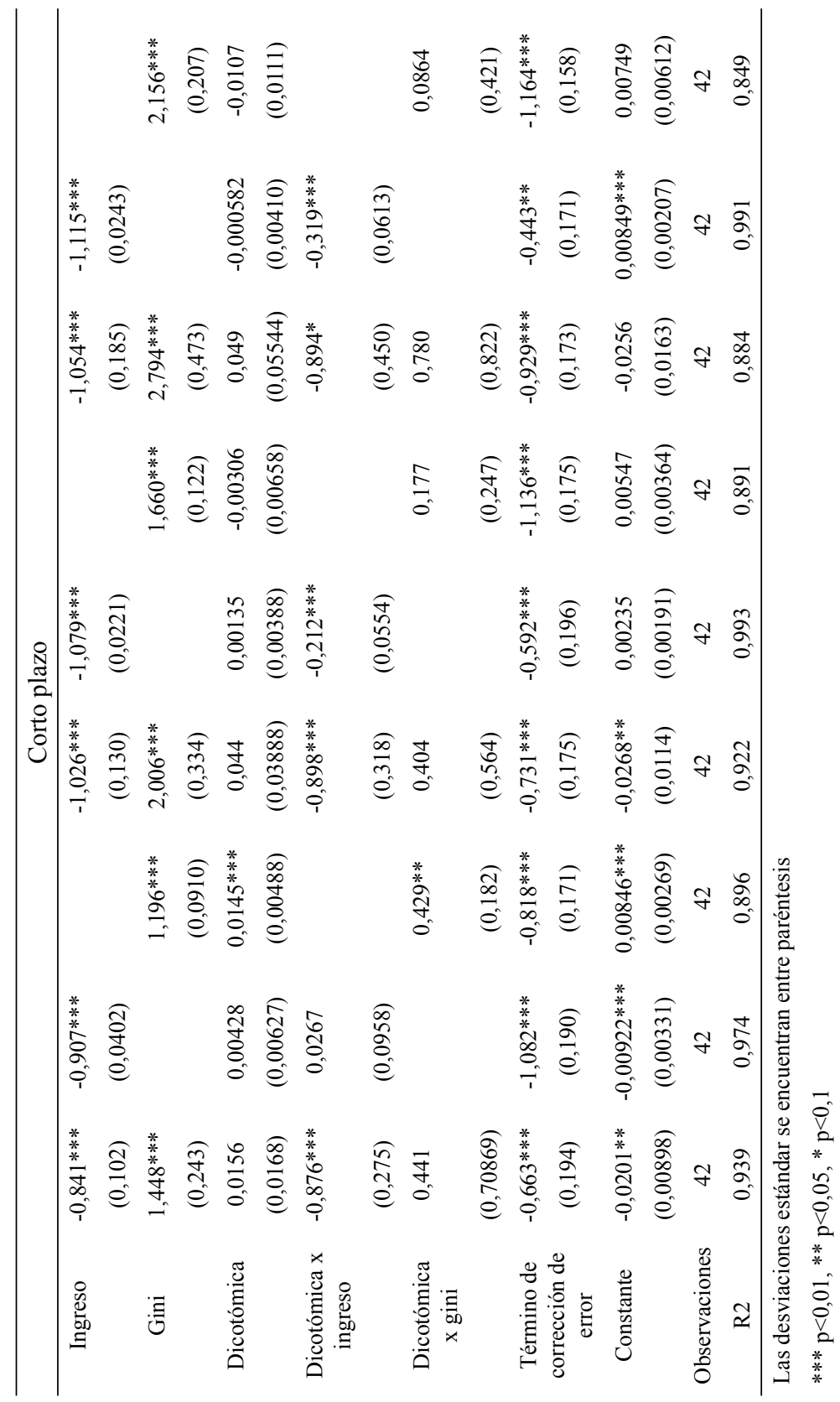


EL EFECTO ASIMETRICO DEL CICLO ECONOMICO SOBRE LAS CONDICIONES SOCIALES...

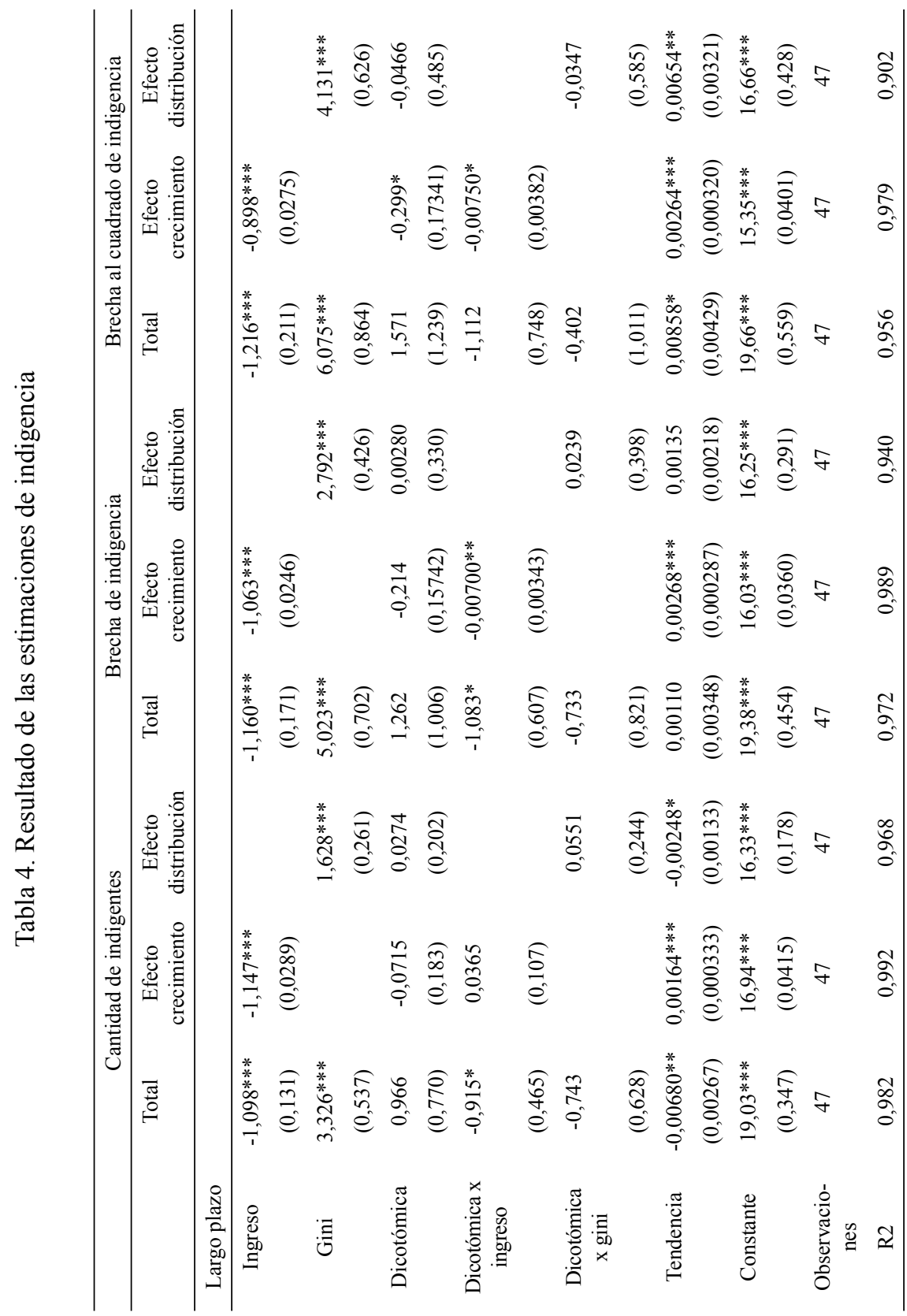




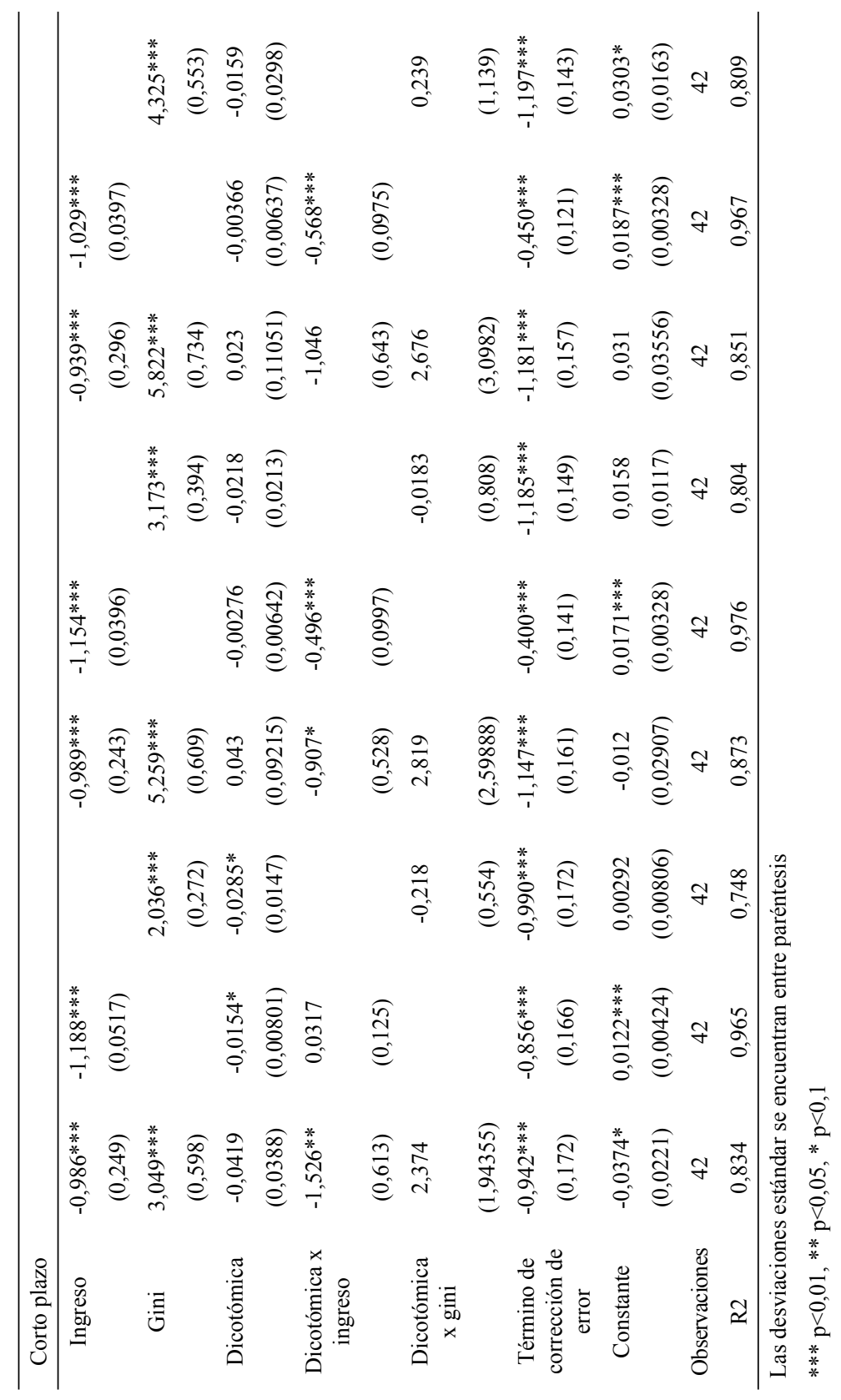




\section{CONCLUSIONES}

Tras la salida del régimen de Convertibilidad, la situación social argentina era alarmante, con más de la mitad de la población sumergida en la pobreza y más de la quinta parte en la indigencia. Pero, a partir de la segunda mitad de 2002, el país comenzó a recuperarse y alcanzó hasta 2011 un fuerte crecimiento económico. Asimismo, esta expansión fue pro-pobre, es decir, no solo permitió la salida de muchas familias de la pobreza y la indigencia, sino que los ingresos de los estratos más bajos fueron los que presentaron un mayor incremento en el período. En efecto, aun siendo un período de elevado aumento del ingreso, fue el efecto distribución el causante principal de las mejoras. Sin embargo, a partir de 2012, el crecimiento y la distribución del ingreso se estancaron y, por lo tanto, las mejoras sociales se interrumpieron e incluso mostraron un leve deterioro. En definitiva, a 2015 el país presenta una situación social preocupante que significa una leve mejora en relación a la década de 1990, pero un claro deterioro en relación a las décadas de 1970 y 1980.

El presente artículo indaga el riesgo de no poder mantener la actual situación social y, menos aún, retomar la senda de la mejora, teniendo en cuenta el menos favorable contexto internacional y la inestable situación cambiaria. Para ello, se analiza la existencia de un efecto asimétrico del ciclo económico sobre las condiciones sociales entre 2003 y 2015 , es decir, que la reducción de la pobreza y la indigencia producto de las observaciones de crecimiento del ingreso fue de menor magnitud que el deterioro en las observaciones de caída. La metodología utilizada consiste en un modelo de corrección de error, en el cual se testea si el valor absoluto de las elasticidades ingreso de la pobreza y de la indigencia es similar en ambos casos.

Los datos de la Argentina entre 2003 y 2015 muestran que la elasticidad ingreso de la pobreza y la indigencia se sitúa en torno a 1, mientras que la elasticidad distribución presenta una mayor dispersión con valores que van de 1,3 a 6. Asimismo, el coeficiente que acompaña al producto del ingreso y la variable dicotómica es significativo en muchos casos y su signo es negativo. Este resultado se corrobora utilizando como variable dependiente tanto la cantidad de pobres e indigentes, como las respectivas brechas y brechas al cuadrado. También se hace como ejercicio de robustez las regresiones desagregando la variación de los indicadores sociales producto, por un lado, del efecto crecimiento y, por otro, distribución del ingreso. En definitiva, hay evidencia que sostiene la existencia del efecto asimétrico del ciclo económico sobre la pobreza y la indigencia durante el período analizado. En otras palabras, al menos entre 2003 y 2015 , un punto porcentual de 
crecimiento económico mejoró las condiciones sociales en menor magnitud de lo que la empeoró un punto de caída en el nivel de actividad ${ }^{10}$.

A la luz de estos resultados, la política macroeconómica más propicia para mejorar las condiciones sociales en forma sostenible no sería aquella que potencia el crecimiento económico de corto plazo, sino la que aboga por una tasa de expansión estable y, especialmente, la que evita las recesiones.

10 Se debe tener en cuenta que a esta conclusión se llegó a partir de una muestra acotada, en la que no se observaron muchas observaciones de caída del ingreso (10 de un total de 42). Es por eso que, si bien los resultados son relevantes, deberían ser corroborados en un horizonte temporal y geográfico más extenso. Se espera poder hacer alguna contribución al respecto en el futuro cercano. 


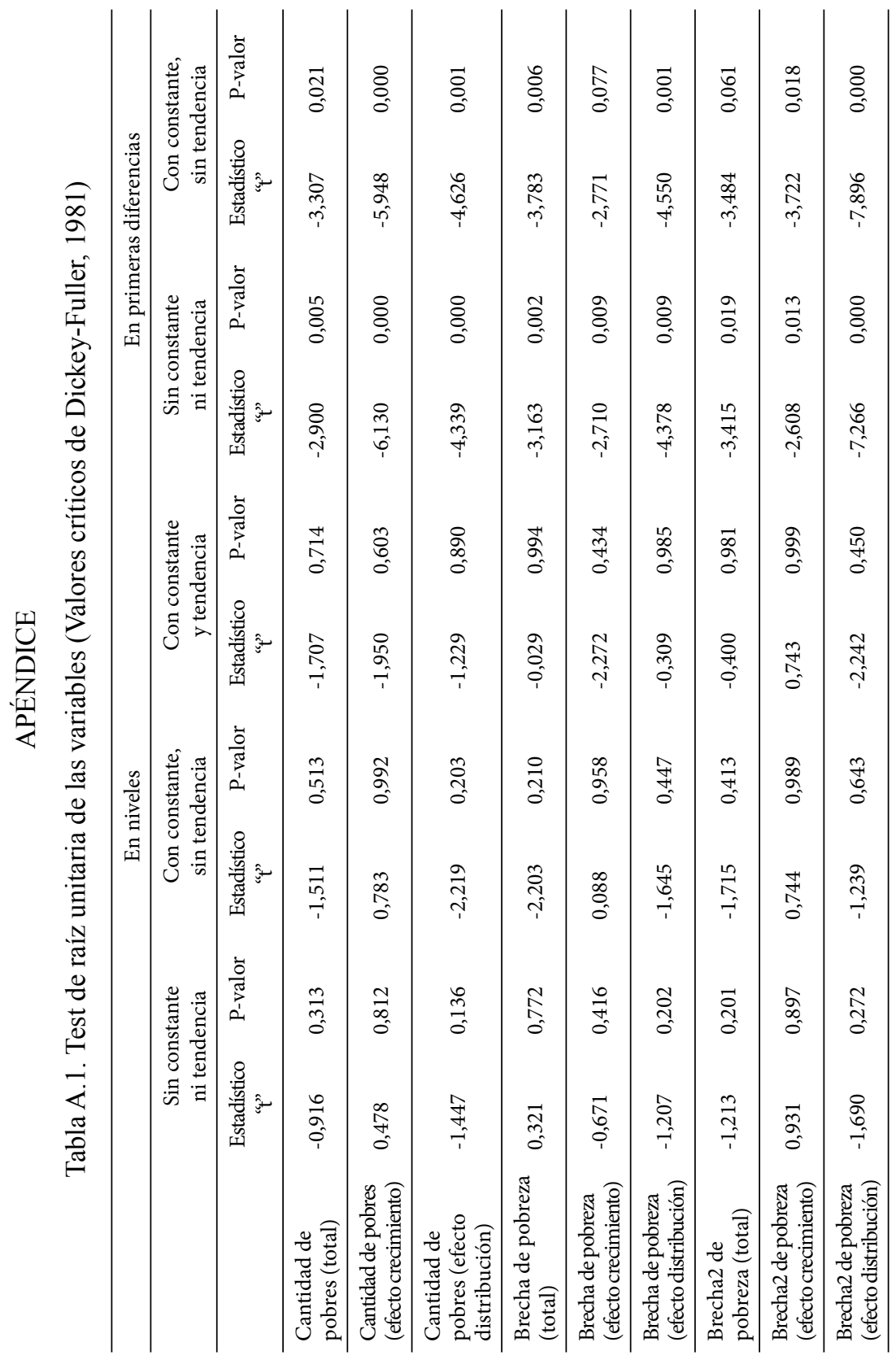




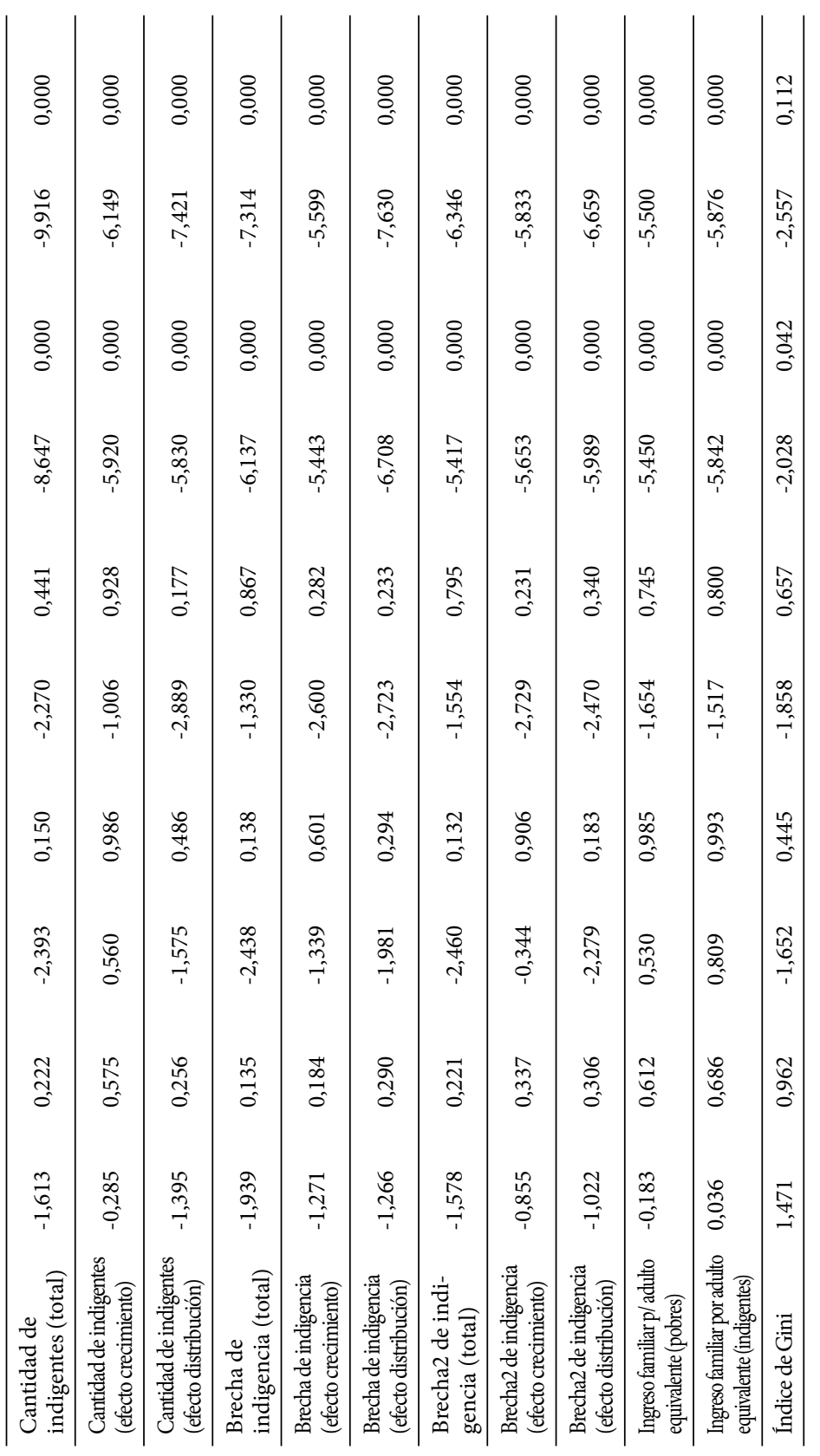


Tabla A.2. Test de raíz unitaria de los residuos de las ecuaciones de largo plazo (Valores críticos de MacKinnon, 1991)

\begin{tabular}{ll}
\hline & Estadístico “t” \\
\hline Pobreza (total) & $-3,472$ \\
Pobreza (efecto crecimiento) & $-4,538$ \\
Pobreza (efecto distribución) & $-4,100$ \\
Brecha de pobreza (total) & $-4,472$ \\
Brecha de pobreza (efecto crecimiento) & $-4,126$ \\
Brecha de pobreza (efecto distribución) & $-6,238$ \\
Brecha2 de pobreza (total) & $-5,506$ \\
Brecha2 de pobreza (efecto crecimiento) & $-3,405$ \\
Brecha2 de pobreza (efecto distribución) & $-7,042$ \\
Indigencia (total) & $-5,294$ \\
Indigencia (efecto crecimiento) & $-6,104$ \\
Indigencia (efecto distribución) & $-4,413$ \\
Brecha de indigencia (total) & $-6,264$ \\
Brecha de indigencia (efecto crecimiento) & $-3,705$ \\
Brecha de indigencia (efecto distribución) & $-4,413$ \\
Brecha2 de indigencia (total) & $-6,205$ \\
Brecha2 de indigencia (efecto crecimiento) & $-6,695$ \\
Brecha2 de indigencia (efecto distribución) & $-7,535$ \\
\hline
\end{tabular}

Valores críticos de MacKinnon: $-4,1378$ ( $\alpha=1 \%),-3,4687(\alpha=5 \%),-3,1354(\alpha=10 \%)$ 


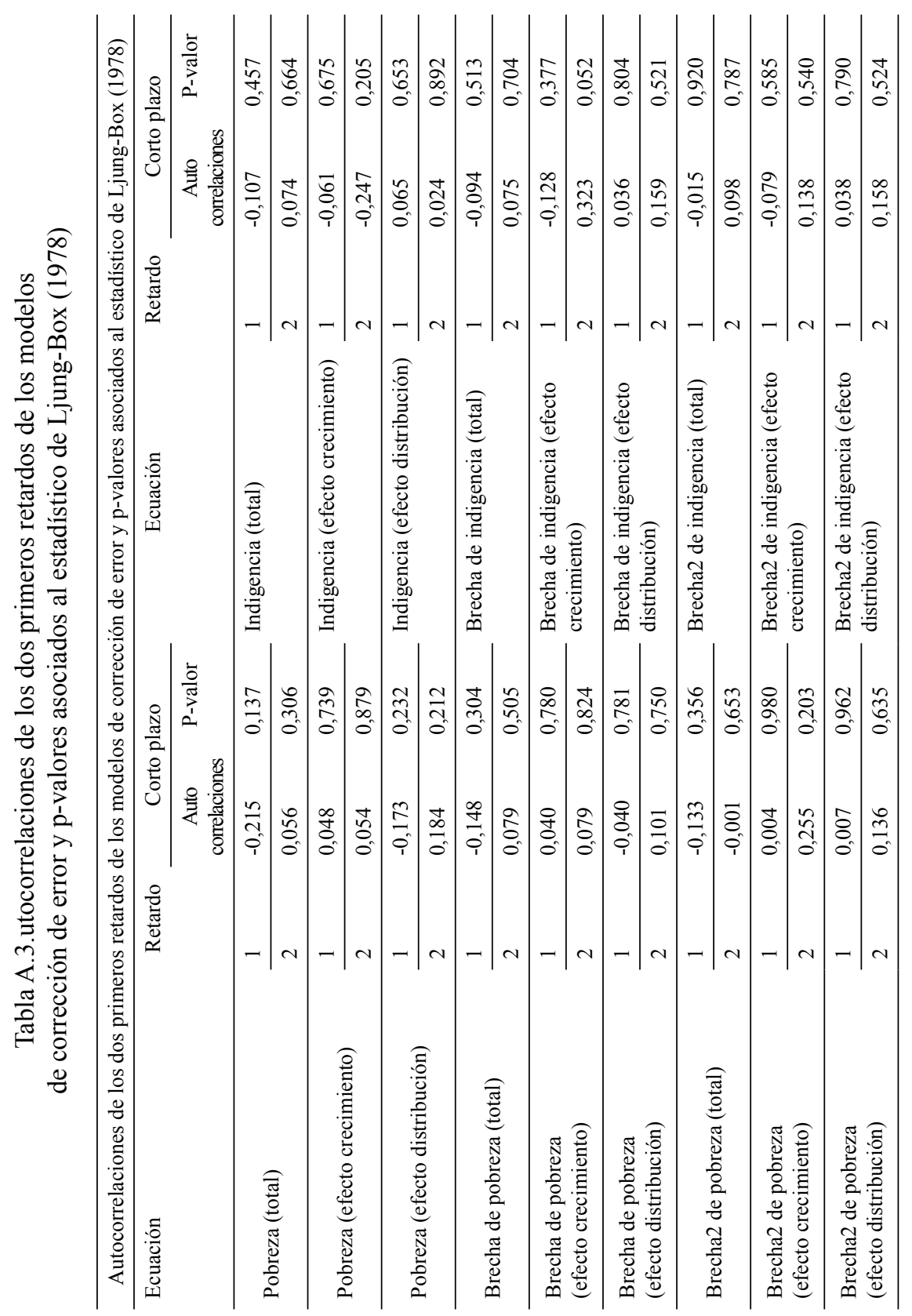




\section{REFERENCIAS BIBLIOGRAFICAS}

Adams, R. H. (2004). Economic growth, inequality and poverty: estimating the growth elasticity of poverty. World Development 32(12), 1989-2014.

Adelman, I., \& Morris, C. T. (1973). Economic growth and social equity in developing countries. Stanford, California: Stanford University Press.

Agénor, P. R. (2004). Macroeconomic adjustment and the poor: analytical issues and cross country Evidence. Journal of Economic Surveys, 18 (3), 351-408.

Ahluwalia, M. S. (1974). Income inequality: some dimensions of the problem. Finance and Development, 11(3), 2.

Ahluwalia, M. S. (1976). Income distribution and development: some stylized facts. The American Economic Review, 66(2), 128-135.

Bhalla, S. S. (2002). Imagine there's no country: Poverty, inequality, and growth in the era of globalization. Washington D. C.: Peterson Institute.

BID (1995). Overcoming Volatility. Economic and Social Progress in Latin America 1995 Report. Washington D.C.: BID.

Bourguignon, F. (2003). The growth elasticity of poverty reduction: explaining heterogeneity across countries and time periods. En T. Eicher \& S. Turnovsky (Eds.). Inequality and growth: Theory and policy implications (pp. 3-26). Massachusetts: MIT Press.

Breen, R. \& García-Peñalosa, C. (2005). Income inequality and macroeconomic volatility: An empirical investigation. Review of Development Economics, 9(3), 380-398.

Bruno, M., Ravallion, M., \& Squire, L. (1998). Equity and growth in developing countries: old and new perspectives on the policy issues. En V. Tanzi \& K. Chu (Eds.). Income distribution and high-quality growth. (pp. 117-146). Cambridge, M.A.: MIT Press.

Busso, M., Cerimedo, F. \& Cicowiez, M. (2005). Pobreza, crecimiento y desigualdad: descifrando la última década en Argentina. Centro de Estudios Distributivos, Laborales y Sociales, Documento de Trabajo No. 21.

Calderón, C., \& Levy Yeyati, E. (2009). Zooming in From Aggregate Volatility to Income Distribution. The World Bank, Policy Research Working Paper No. 4895.

Cline, W. R. (1975). Distribution and development: A survey of literature. Journal of Development Economics, 1(4), 359-400.

Datt, G., \& Ravallion, M. (1992). Growth and redistribution components of changes in poverty measures: A decomposition with applications to Brazil and India in the 1980s. Journal of development economics, 38(2), 275-295.

De Janvry, A., \& Sadoulet, E. (2000). Growth, poverty, and inequality in Latin America: A causal analysis, 1970-94. Review of Income and Wealth, 46(3), 267-287. 
Dickey, D. A., \& Fuller, W. A. (1981). Likelihood ratio statistics for autoregressive time series with a unit root. Econometrica, 49 (4), 1057-1072.

Fang, W., Miller, S. M., \& Yeh, C. C. (2015). The effect of growth volatility on income inequality. Economic Modelling, 45, 212-222.

Fields, G. S. (1980), Poverty, Inequality, and Development. New York: Cambridge University Press.

Fields, G. S. (1989). Changes in poverty and inequality in developing countries. The World Bank Research Observer, 4(2), 167-185.

Gasparini, L., Gutiérrez, F., \& Tornarolli, L. (2007). Growth and income poverty in Latin America and the Caribbean: evidence from household surveys. Review of Income and Wealth, 53(2), 209-245.

Gasparini, L., Cicowiez, M. \& Escudero, W. S. (2013). Pobreza y desigualdad en América Latina. Conceptos, herramientas y aplicaciones. Buenos Aires: Temas.

Hausmann, R., \& Gavin, M. (1996). Securing Stability and Growth in a Shock Prone Region: The Policy Challenge for Latin America. Inter-American Development Bank, Working Paper No. 315.

Kravis, I. (1960). International Differences in the Distribution of Income. Review of Economics and Statistics, 42(4), 408-416.

Kuznets, S. (1955). Economic Growth and Income Inequality. The American Economic Review, 45(1), 1-28.

Laursen, T., \& Mahajan, S. (2004). Volatility, income distribution, and poverty. En J. Aizenman, \& B. Pinto Managing (Eds). Volatility and crises. A practitioner guide (pp. 101-136). Cambridge: Cambridge University Press.

Ljung, G. M., \& Box, G. E. P. (1978). On a measure of a lack of fit in time series models. Biometrika, 65(2), 297-303.

Locke Anderson, W. H. (1964). Trickling down: the relationship between economic growth and the extent of poverty among american families. The Quarterly Journal of Economics, 78(4), 511-524.

Lustig, N. (2000). Crises and the Poor: Socially responsible macroeconomics. Inter-American Development Bank, Technical Papers Series No. POV-108.

MacKinnon, J. G. (1991). Critical Values for Cointegration Tests. En R. F. Engel ,\& C. Granger (Eds.). Long Run Economic Relationship. (pp. 267-276). Oxford: Oxford University Press.

Mahmoudi, V. (mayo, 2001). Growth-equity decomposition of a change in poverty: an application to Iran. Trabajo presentado en UNU/WIDER Development conference on growth and poverty, Helsinki, Finlandia.

Medina, F. \& Galván, M. (2014). Crecimiento económico, pobreza y distribución del ingreso: Fundamentos teóricos y evidencia empírica para América Latina, 1997-2007. Serie Estudios Estadísticos No. 82, Santiago de Chile: CEPAL. 
Oshima, H. (1970). Income inequality and economic growth: The post-war experience of Asian countries. Malayan Economic Review, 15, 7-41.

Partridge, M. D., Rickman, D. S., \& Levernier, W. (1996). Trends in U.S. income inequality: evidence from a panel of states. The Quarterly Review of Economics and Finance, 36(1), 17-37.

Paukert, F. (1973). Income Distribution at Different Levels of Development: A Survey of Evidence. International Labour Review, 108, 97.

Ram, R. (1991). Kuznets's inverted-U hypothesis: evidence from a highly developed country. Southern Economic Journal, 57(4), 1112-1123.

Ravallion, M., \& Chen, S. (1997). What can new survey data tell us about recent changes in distribution and poverty?. The World Bank Economic Review, 11(2), 357-382.

Robinson, S. (1976). A note on the $\mathrm{U}$ hypothesis relating income inequality and economic development. The American Economic Review, 66, (3), 437-440.

(C) 2016 por los autores; licencia otorgada a la Revista Estudios Económicos. Este artículo es de acceso abierto y distribuido bajo los términos y condiciones de una licencia Atribución-No Comercial 3.0 Unported (CC BY-NC 3.0) de Creative Commons. Para ver una copia de esta licencia, visite http://creativecommons.org/licenses/by-nc/3.0/ 
